\title{
Rotation and density asymmetries in the presence of large poloidal impurity flows in the edge pedestal
}

\author{
E. Viezzer ${ }^{1}$, T. Pütterich ${ }^{1}$, E. Fable ${ }^{1}$, A. Bergmann ${ }^{1}$, R. Dux ${ }^{1}$, \\ R. M. McDermott ${ }^{1}$, R. M. Churchill ${ }^{2}$, M. G. Dunne ${ }^{3}$ and the \\ ASDEX Upgrade Team \\ ${ }^{1}$ Max-Planck-Institut für Plasmaphysik, EURATOM Association, Boltzmannstr. 2, \\ 85748 Garching, Germany \\ ${ }^{2}$ MIT Plasma Science and Fusion Center, Cambridge, USA \\ ${ }^{3}$ Department of Physics, University College Cork, Association Euratom-DCU, Cork, \\ Ireland \\ E-mail: Eleonora.Viezzer@ipp.mpg.de
}

\begin{abstract}
Novel flow rotation measurements based on charge exchange recombination spectroscopy at the inboard midplane of the ASDEX Upgrade tokamak reveal the existence of an asymmetric flow structure at the H-mode edge, which is shown to arise due to a poloidal impurity density asymmetry. A quantitative evaluation of the impurity density at the inboard side demonstrates that the impurities redistribute along the flux surface, resulting in a poloidal dependency of the impurity density. The poloidal and toroidal impurity flows measured at the high-field side (HFS) and low-field side (LFS) are compared to theoretical predictions based on the parallel momentum balance which includes friction, inertia, the pressure and the electric force. Both a fluid and a kinetic approach are used showing good agreement between each other. The measured impurity flow structure is described by the model quantitatively when a finite poloidal main ion flow of $\sim 2 \mathrm{~km} / \mathrm{s}$ arises, which is in keeping with the standard neoclassical prediction. The interplay of all terms, in particular the inclusion of the impurity inertia term, is important to reproduce the observed flow structure and results in an impurity accumulation at the HFS. The existence of a poloidal impurity density asymmetry in the edge transport barrier slightly reduces the drift parameter $v / D$, however, the experimental value is consistent with standard neoclassical theory. This demonstrates that despite the asymmetry in the impurity density, the impurity particle transport is at the neoclassical level.
\end{abstract}

PACS numbers: 52.55.Fa, 52.25.Vy, 52.30.-q, 52.70.-m

\section{Introduction}

The interplay between macroscopic flows and transport is of crucial importance to the understanding of plasma confinement and stability. In tokamaks, transport is observed to be dominated by anomalous effects [1], however, there is growing evidence that under certain conditions the ion transport can be explained by neoclassical theory. In the edge 
transport barrier of $\mathrm{H}$-mode plasmas, where turbulent transport is strongly reduced, the ion heat transport level and the impurity particle transport have been observed to be near neoclassical values [2].

The poloidal rotation velocity is of particular interest for its role in two phenomena: parallel viscous momentum transport and the shear-flow turbulence-suppression feedback loop, which is responsible for access to the H-mode operation regime. In standard neoclassical theory, the poloidal rotation is coupled to poloidal density asymmetries via particle and momentum conservation. Such poloidal asymmetries can be generated by, amongst others, neoclassical effects [3] or a poloidal variation of the impurity source location $[4,5]$. Previous studies have shown that friction between main ions and impurities, which arises due to steep temperature and density gradients, might be one possible mechanism that gives rise to a poloidal impurity density asymmetry $[6,7,8]$. Rigid toroidal plasma rotation has also been considered as one possible player $[9,10,11]$; here, the centrifugal force leads to an impurity accumulation at the outboard side. In addition, in-out asymmetries in the plasma core can arise when ion cyclotron radio frequency heating is applied $[12,13]$, which can generate an outward convection of the impurities $[14,15]$.

Poloidal impurity density asymmetries have been observed in the plasma core $[12,16$, $17,18]$ as well as at the edge $[19,20,21]$. Especially for the edge transport barrier with its steep temperature and density gradients the dominating mechanism responsible for the existence of an asymmetric impurity density profile is ambiguous and a quantitative interpretation has so far not been provided.

Here, the question of what causes the flow structure (and in turn the structure of the impurity particle density) in the edge pedestal of a confined tokamak plasma is adressed. In this paper we present the first measurements of poloidal and toroidal impurity ion rotation velocities at the high-field side (HFS) and low-field side (LFS) of the ASDEX Upgrade (AUG) tokamak. The flow structure is measured using active charge exchange recombination spectroscopy (CXRS) [22] and compared to theoretical predictions based on the parallel momentum balance equation, which includes inertia, friction, the pressure and electric force [23]. The simulations are performed using a fluid model [23] and the drift-kinetic particle code HAGIS [24, 25, 26]. Both the poloidal and toroidal impurity flows and the poloidal impurity density asymmetry are well described by the theoretical predictions and, for the first time, the observed flow structure is reproduced quantitatively. The main drive for the impurity density asymmetry is given by the friction force, while towards the last closed flux surface the poloidal centrifugal term also gives a substantial contribution.

This paper is structured as follows: section 2 describes the measurement technique and presents H-mode edge rotation profiles measured at the HFS and LFS. In section 3 the evaluation of the impurity density at the HFS is presented. Section 4 discusses the comparison of the experimental data to the theoretical predictions and to kinetic simulations. In section 5 the impact of the findings presented with respect to impurity transport are assessed and a summary and conclusions are given in section 6 . 


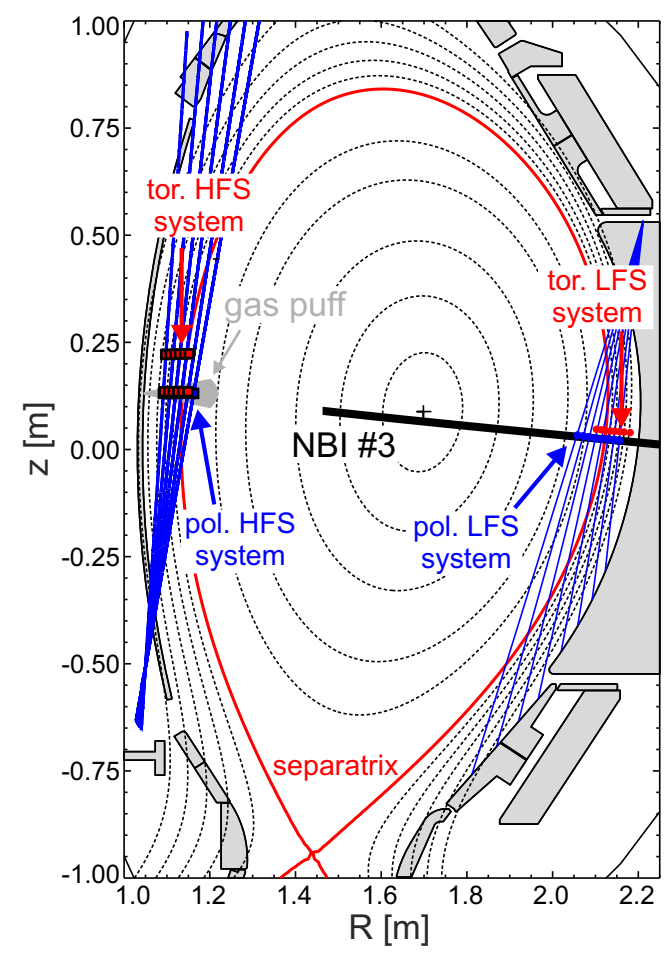

Figure 1: Poloidal cross-section of AUG indicating the measurement locations of the edge CXRS diagnostics.

\section{Rotation measurements at the inboard and outboard midplane of AUG}

At ASDEX Upgrade (AUG) the edge rotation velocities of impurity ions are measured at the low-field side (LFS) and high-field side (HFS) with four independent diagnostics. The LFS system is based on charge exchange recombination spectroscopy (CXRS) at a neutral beam [27] and consists of a toroidal and a poloidal view (see figure 1). The HFS diagnostics utilize a diagnostic D gas puff, and collect light that is emitted after the impurity species undergoes a charge exchange $(\mathrm{CX})$ reaction with a thermal D particle. Using the gas puff technique [28, 29], CXRS measurements are obtained only in the outermost region of the confined plasma since the neutral density, and thus the CX signal, drops rapidly due to the low energy of the injected thermal D atoms. The HFS array consists of a toroidal [20] and a poloidal view (see figure 1) and each view is equipped with two $f / 4$ optical heads to allow for background subtraction (see [28] for details on the experimental setup).

The HFS measurements combined with the data obtained from the LFS diagnostics enable localized CXRS measurements at two different poloidal locations on a flux surface. At the plasma edge the rotation velocities are low and, therefore, accurate knowledge of the wavelength calibration is needed. The wavelength calibration is performed on a shot-to-shot basis using a neon lamp [27]. This method allows the determination of the wavelength calibration quite accurately with uncertainties of the order of $\sim 1 \mathrm{~km} / \mathrm{s}$. 


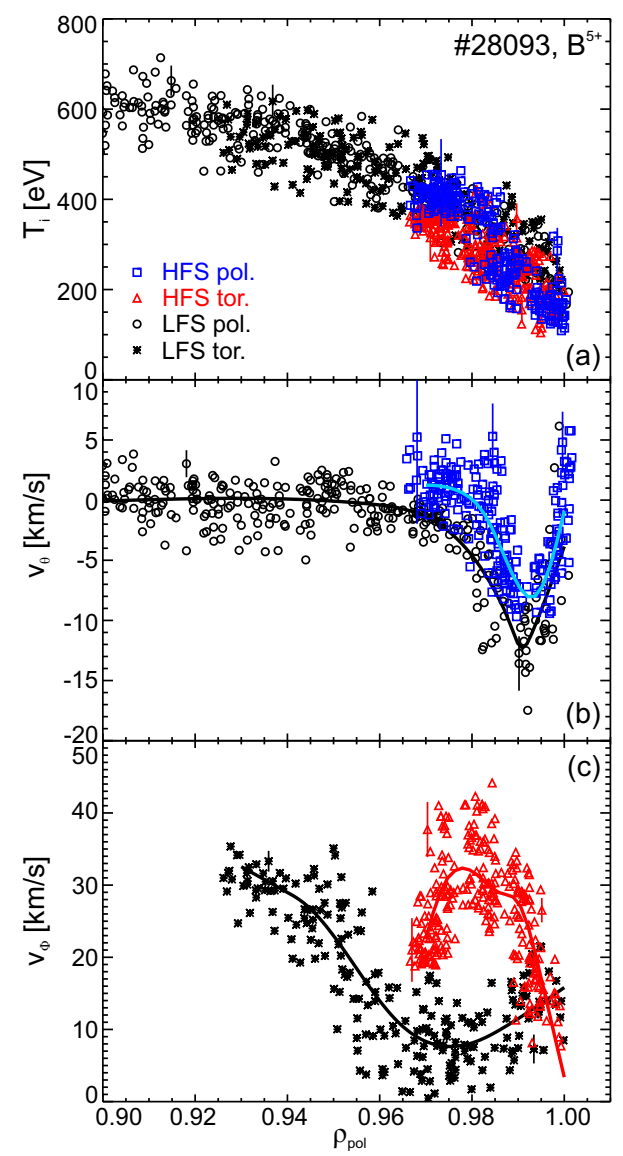

Figure 2: CXRS measurements at the HFS and LFS: (a) $T_{i}$, (b) poloidal rotation and (c) toroidal rotation of $\mathrm{B}^{5+}$. Data obtained with the LFS systems are shown in black, while measurements from the HFS systems are shown in blue (poloidal view) and red (toroidal view). For better clarity the uncertainties of the measurements are shown at distinct radial positions.

Figure 2 shows example profiles of the ion temperature $\left(T_{i}\right)$, poloidal and toroidal impurity rotation velocities $\left(v_{\theta, \alpha}\right.$ and $\left.v_{\phi, \alpha}\right)$ obtained at the LFS and HFS. Toroidal rotation velocities, that are counter-clockwise as viewed from above, are positive, while poloidal rotation velocities, that are vertically upward at the LFS, are negative (electron diamagnetic drift direction). The profiles were measured on $\mathrm{B}^{5+}(n=7 \rightarrow 6, \lambda=$ $494.467 \mathrm{~nm}$ ) in an $\mathrm{H}$-mode discharge with a toroidal magnetic field on-axis of $-2.5 \mathrm{~T}$, plasma current of $1 \mathrm{MA}, 5 \mathrm{MW}$ of neutral beam injection (NBI) heating and $1.5 \mathrm{MW}$ electron cyclotron resonance heating. In this discharge the main ion collisionality at the pedestal top was approximately 2, i.e. in the plateau regime, while the impurities are deep in the Pfirsch-Schlüter regime. In the standard magnetic configuration of AUG $B_{\phi}$ is negative (clockwise viewed from above) and $B_{\theta}$ is positive (pointing downward at the outer midplane). The plasma current and the NBI are pointing into the positive toroidal direction, i.e. counter-clockwise viewed from above. The toroidal rotation velocities shown in figure 2(c) are co-current. 
To obtain detailed radial CXRS profiles of both the HFS and LFS the plasma is radially moved through the views of the LOS [27] during a steady state phase of the discharge. First, the plasma is moved over $700 \mathrm{~ms}$ towards the outer wall to measure complete LFS profiles and then towards the inner wall to obtain full radial HFS profiles. Shortly before the plasma is moved towards the inner wall the diagnostic gas puff is switched on. At the LFS, the alignment of the toroidal and poloidal CXRS measurements with respect to the separatrix position is performed via the $T_{i}$ and impurity density $\left(n_{\alpha}\right)$ profiles to reduce uncertainties due to the magnetic equilibrium [30]. The HFS measurements are then aligned relative to the LFS measurements via the $T_{i}$ profile assuming that $T_{i}$ is constant along the flux surface [7]. The accuracy of the relative profile alignment is estimated to be $2-3 \mathrm{~mm}[30]$.

After aligning the HFS profiles with respect to the LFS profiles, the following flow structure is observed: Inside the ETB the poloidal impurity flow exhibits a strongly sheared rotation in the electron diamagnetic drift direction both at the LFS and HFS with the minimum in $v_{\theta, \alpha}$ approximately at the same radial position. The HFS poloidal rotation velocity is about a factor of 1.5-2 lower than at the LFS. The toroidal rotation velocity is co-current at both the LFS and HFS, however, the profile exhibits an asymmetric structure [20]. At the LFS the toroidal rotation velocity exhibits a minimum located around the pedestal top [31], while at the HFS the profile shows the opposite behaviour and exhibits a maximum at this position (cf. figure 2(c)). Towards the separatrix the toroidal flow at the LFS increases, while it decreases at the HFS.

Both the toroidal flow asymmetry and the difference in magnitude of the poloidal flow might be explained by an excess of impurity density at the HFS, as postulated in [19, 20] and observed in Alcator C-Mod [21]. A poloidal impurity density asymmetry can be inferred based on the neoclassical formalism for the total flow on a flux surface [19, 20]. Allowing for a poloidal dependence of the impurity density, the lowest order divergencefree flow of a species $\alpha$ on a flux surface is characterized by [32, 33]:

$$
\mathbf{v}_{\alpha}=\omega_{\alpha}(\Psi) R \mathbf{e}_{\phi}+\frac{k_{\alpha}(\Psi)}{n_{\alpha}} B \mathbf{e}_{\|}
$$

where $\omega_{\alpha}(\Psi)$ and $k_{\alpha}(\Psi)$ are flux functions, $\Psi$ is the poloidal magnetic flux, $R$ the local major radius, $\mathbf{e}_{\phi}$ and $\mathbf{e}_{\|}$the unit vectors in the toroidal and parallel direction, $n_{\alpha}$ the impurity density and $B$ the magnetic field. The divergence-free flow on a flux surface is a composite of the rigid body rotation (first term on the right-hand side of equation (1)) and the parallel flow. Assuming that the impurity density is a flux function, $k_{\alpha}(\Psi)$ would result in $k_{\alpha}(\Psi)=\frac{v_{\theta, \alpha}}{B_{\theta}}$ and thus, $v_{\theta, \alpha}$ is expected to scale with $B_{\theta}$, which is higher at the HFS. Figure 2(b) shows that this dependence is violated since the HFS poloidal rotation is smaller than at the LFS.

Figure 3 shows the measured parallel and poloidal velocity at the HFS (solid black lines). The differences between parallel and toroidal flows are small due to the geometry of the magnetic field lines. However, these differences are taken into account in the following and the measured parallel (and poloidal) impurity flows are compared to the theoretical predictions. The red, dashed lines in figure 3 show the expected parallel and poloidal 


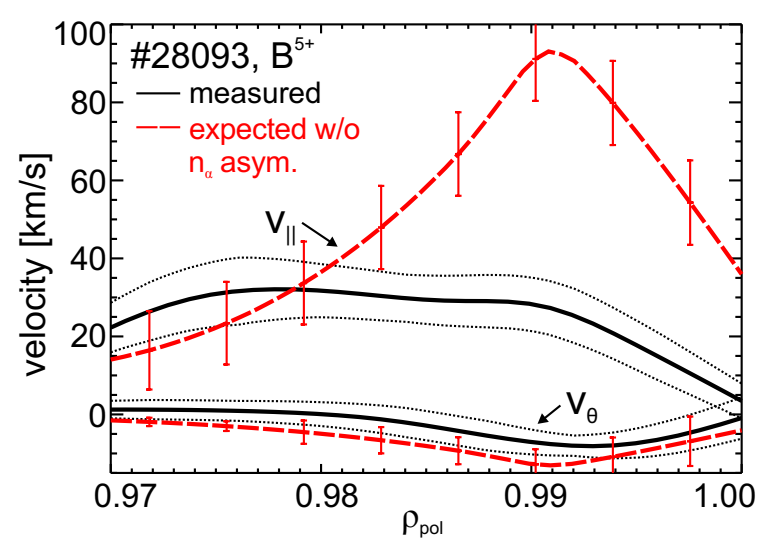

Figure 3: Parallel and poloidal velocities at the HFS: the measured profiles are shown in black while the expected profiles without the existence of an impurity density asymmetry are shown in red (dashed lines). The dotted black lines indicate the uncertainties in the measurement.

impurity flows if no impurity density asymmetry existed. These profiles have been evaluated from the measured LFS profiles using equation (1), assuming that the impurity density is constant on a flux surface. As shown in figure 3 the expected velocities are up to a factor of $\sim 4(\sim 2)$ too high for the parallel (poloidal) flow. This indicates that a poloidal impurity density asymmetry is present in the edge transport barrier. If a poloidal impurity density asymmetry exists $[19,20]$ the condition of divergence-free flows can be fulfilled.

\section{Analysis of HFS impurity density and comparison to the LFS}

\subsection{Direct evaluation of the impurity density from the measured Balmer spectral radiance}

For usual CXRS applications the impurity density is evaluated from the measured radiance of the CX signal observed by the line of sight (LOS) combined with knowledge of the neutral density profile and the charge exchange cross-sections. For thermal neutrals the radiance (in photons $/ \mathrm{sr} / \mathrm{m}^{2} / \mathrm{s}$ ) is given by:

$$
L_{C X}^{p h}(\lambda)=\frac{1}{4 \pi} \int_{L O S} \sum_{n=1}^{n_{\max }} n_{\alpha} n_{\mathrm{D}, n}\left\langle\sigma_{n} v\right\rangle_{\mathrm{eff}, \lambda} d l
$$

where $n_{\alpha}$ is the density of the impurity $\alpha, n_{\mathrm{D}, n}$ the neutral density with main quantum number $n$ and $\left\langle\sigma_{n} v\right\rangle_{\mathrm{eff}, \lambda}$ the effective $\mathrm{CX}$ rate coefficient, which is derived from the Atomic Data Analysis Structure (ADAS) database [34]. 〈〉 indicates the Maxwellian average over the cross-section $\sigma$ and the relative velocity $v$ between the reaction partners, i.e. $v=\left|\mathbf{v}_{\mathrm{D}, n}-\mathbf{v}_{\alpha}\right|$ with $\mathbf{v}_{\mathrm{D}, n}$ being the velocity of the neutral and $\mathbf{v}_{\alpha}$ the velocity of the impurity. Here, the integration is along the LOS and the contributions from all states of the deuterium neutrals (ground and excited states) are summed up. 


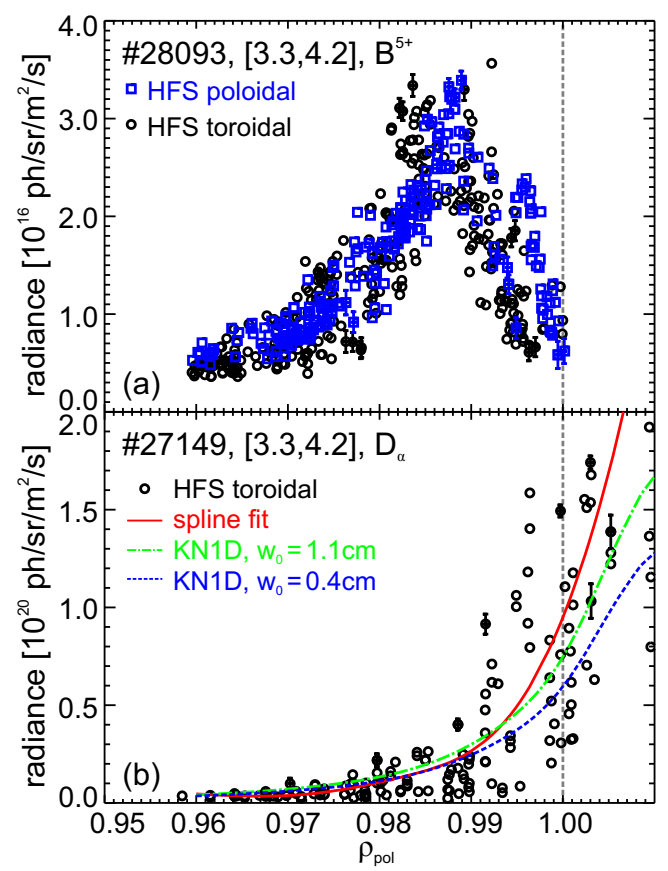

Figure 4: Measured spectral radiance of (a) $\mathrm{B}^{5+}$ and (b) $\mathrm{D}_{\alpha}$. Note the difference in magnitude when comparing the absolute values. In (b) the radiance profiles as simulated with KN1D are shown in green (dashed-dotted line) and blue (dashed line).

For charge exchange with thermal neutrals the dominant contribution is given by thermal neutral $\mathrm{D}$ in the $n=2$ excited state $[28,35]$. At low collision energies and considering the $\mathrm{B}^{5+}$ transition, the $\mathrm{CX}$ cross-section for $\mathrm{D}$ in $n=1(n=3)$ is six (four) orders of magnitude smaller than for $\mathrm{D}$ in $n=2$ [28]. Hence, assuming that the impurity density is constant in the volume where the LOS crosses the gas puff, the impurity density $n_{\alpha}$ can be evaluated as follows:

$$
\begin{aligned}
L_{C X}^{p h}(\lambda) & =\frac{1}{4 \pi} n_{\alpha}\left\langle\sigma_{n=2} v\right\rangle_{\mathrm{eff}, \lambda} \int_{L O S} n_{\mathrm{D}, n=2} d l \\
& =\frac{1}{4 \pi} n_{\alpha}\left\langle\sigma_{n=2} v\right\rangle_{\mathrm{eff}, \lambda} \frac{f_{n=2}}{f_{n=3}} \frac{1}{A_{32}} \int_{L O S} \varepsilon_{32} d l \\
\Rightarrow n_{\alpha} & =\frac{A_{32}}{\left\langle\sigma_{n=2} v\right\rangle_{\mathrm{eff}, \lambda}} \frac{f_{n=3}}{f_{n=2}} \frac{L_{C X}^{p h}}{L_{32}^{p h}}
\end{aligned}
$$

Here, $A_{32}$ is the Einstein coefficient for the Balmer $(n=3 \rightarrow 2)$ transition, $f_{n=2}$ and $f_{n=3}$ the fractional abundances of thermal neutral $\mathrm{D}$ in the $n=2$ and $n=3$ excited states, and $L_{32}$ the line-integrated emissivity of the $D_{\alpha}$ spectral line. Hence, the impurity density can be derived from the measured spectral radiance of the CX line and the $D_{\alpha}$ spectral line. Figure 4 shows (a) the measured spectral radiance of $\mathrm{B}^{5+}$ as measured with the poloidal and toroidal HFS system and (b) the $D_{\alpha}$ radiance measured in a discharge that is identical to the one described in section 2. Here, only data from the toroidal HFS view is shown as the poloidal system was not yet available. In addition, the spectral radiance derived from the neutral emissivity as simulated with KN1D (see 
next subsection) is shown in figure 4(b).

\subsection{Calculation of $n_{\alpha}$ from simulated neutral density profiles}

The impurity density can also be evaluated from modelling of the gas puff penetration. The neutral density profiles have been simulated with the 1D in space and 2D in velocity kinetic transport model KN1D [36]. The code uses a slab-like spatial geometry and calculates the distribution functions of neutral atomic and molecular deuterium using a method of successive collision generations with specified input plasma parameters. The molecular neutral pressure at the wall, which is used as input for KN1D, is derived from the manometer measurement of the gas reservoir during the $\mathrm{D}_{2}$ injection. To obtain the actual $\mathrm{D}_{2}$ injection rate from the manometer measurement a conversion factor is applied that has been evaluated from a dedicated two chamber experiment [37]. From this injection rate the molecular neutral pressure is calculated taking a cross-sectional area of the gas puff cloud into account, i.e. the molecular neutral pressure is scaled such that the resulting neutral densities fit approximately to the profile derived from the $\mathrm{D}_{\alpha}$ measurement.

In [28] the shape and the particle density of the gas puff cloud was studied in detail using OSM-EIRENE simulations. It was found that the shape of the gas puff stays approximately the same despite different plasma conditions. The shape of the gas puff is well described by a diverging Gaussian beam [28], $n_{D}(R, x)=n_{D, 0}(R) \exp \left(-(x / w(R))^{2}\right)$, where $x$ is along the line of sight, $n_{D, 0}(R)$ is the nominal neutral density along the center of the gas puff and $w(R)=w_{0}+d(R) \tan (\theta / 2)$ the width of the Gaussian. Here, $w_{0}$ corresponds to an apparent beam width at the gas puff location (if the shape of the gas puff resembled a truncated cone), $d(R)$ is the distance to the gas puff location, and $\theta$ the opening angle of the gas puff [28]. Due to the very similar setup of the gas valve at CMod and AUG (horizontal injection), the same opening angle of $\theta \approx 46^{\circ}$ is used for the simulated profiles. However, the value for $w_{0}$ might change depending on the distance between the measurement volume (as seen by the lines of sight of the diagnostics) and the location of the gas puff. At AUG, the distance between the gas puff location and the separatrix is typically $\sim 6 \mathrm{~cm}$. For the KN1D simulations presented here, $w_{0}=0.4 \mathrm{~cm}$ was used which corresponds to the inner diameter of the gas valve. For a comparison, the value stated in [28], $w_{0}=1.1 \mathrm{~cm}$, was used as well. It should be noted, however, that the choice of $w_{0}$ does not have a significant impact on the shape of the resulting impurity density profile, but rather on the absolute magnitude (see figure 5).

From the neutral density $n_{D, 0}$ profiles as simulated with KN1D the impurity density at the HFS is derived by taking the geometry of the gas puff into account. Figure 5 shows the impurity density profile at the LFS in black and colour-coded at the HFS. The profile at the LFS has been evaluated including the effect due to the beam halo $[38,39]$, which is produced by charge transfer from beam neutrals to deuterium ions. The red HFS profile corresponds to the evaluation using the measured Balmer spectral radiance and the profiles shown in blue and green correspond to the simulated $n_{D}$ profiles 


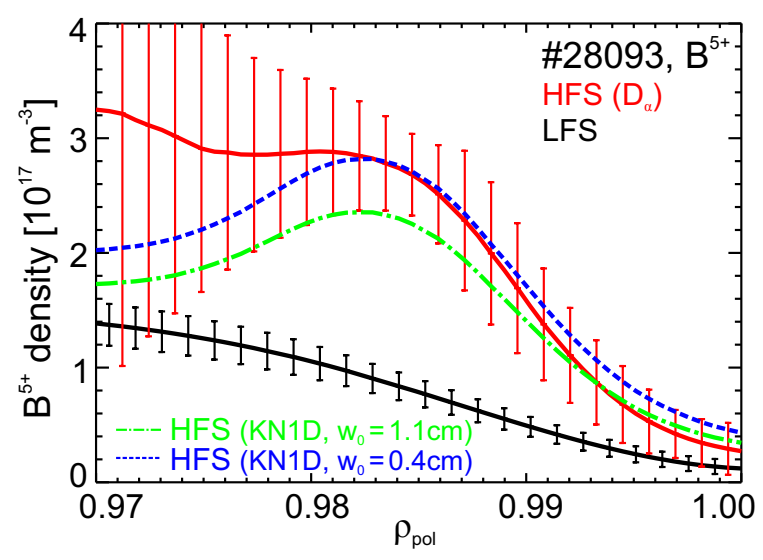

Figure 5: $\mathrm{B}^{5+}$ density at the LFS (black) and HFS (colour-coded): The red profile has been evaluated using the measured radiance of the Balmer spectral line, while the profiles calculated using the neutral density profiles simulated with KN1D are shown in blue $\left(w_{0}=0.4 \mathrm{~cm}\right.$, dashed line $)$ and green $\left(w_{0}=1.1 \mathrm{~cm}\right.$, dashed-dotted line).

with $w_{0}=0.4 \mathrm{~cm}$ and $w_{0}=1.1 \mathrm{~cm}$, respectively. As shown in figure 5 the impurity density is asymmetric on a flux surface, with an impurity accumulation at the HFS. Comparing the HFS impurity density profile as derived from the $\mathrm{D}_{\alpha}$ spectral line to the one evaluated using the KN1D simulation shows a relatively good agreement for the region with a negative radial gradient. Further inwards $\left(\rho_{\text {pol }}<0.98\right)$, the shape deviates. Note, however, that towards the plasma core the uncertainties in the HFS measurements increase due to the limited penetration of the gas puff neutrals. In addition, since KN1D is a $1 \mathrm{D}$ code and $3 \mathrm{D}$ effects might affect the total shape of the neutral density (and thus, the resulting $n_{\alpha}$ profile), the KN1D simulation also has uncertainties in the input values. In the region with the maximum asymmetry (see also figure 8) both methods fit reasonably well with one another.

Based on these measurements there is direct evidence that the impurity density is asymmetric on the flux surfaces. Thus, the total flow on a flux surface is described by equation (1) where the parallel flow (second term on the right-hand side of equation (1)) depends on the impurity density which has poloidal variations along the flux surface. In the next section, the possible mechanism that could generate a poloidal impurity density asymmetry inside the edge transport barrier, which in turn affects the flow structure on the flux surfaces, is presented.

\section{Comparison to theoretical predictions}

The measurements of the rotation velocities at both the LFS and HFS enable a detailed study of the flow structure at the edge of a tokamak plasma. In particular, the measured rotation velocities at two different points along the poloidal arc allow for a comparison to calculations based on neoclassical theory [23]. The poloidal impurity distribution is determined by the parallel momentum balance equation which includes inertia, both 
the pressure and electric drive and friction [23]:

$\mathbf{B} \cdot \nabla\left[\frac{k_{\alpha}^{2} B^{2}}{2 n_{\alpha}^{2}}-\frac{R^{2} \omega_{\alpha}^{2}}{2}+v_{t h, \alpha}^{2}\left(\log \left(n_{\alpha}\right)+\frac{Z_{\alpha} \tilde{\Phi}}{T_{\alpha}}\right)\right]+\nu_{\alpha i} B\left(u_{\|, \alpha}-u_{\|, i}+\Delta Q_{\|}\right)=0$.

Here, $\mathbf{B}$ is the magnetic field, $k_{\alpha}$ is a flux function, $n_{\alpha}$ is the density of the impurity species $\alpha, R$ is the local major radius, $\omega_{\alpha}$ is the rigid rotation velocity defined as $\omega_{\alpha}=\frac{1}{R B_{\theta}}\left(E_{r}-\nabla p_{\alpha} /\left(n_{\alpha} Z_{\alpha} e\right)\right)$ where $E_{r}$ is the radial electric field and $\nabla p_{\alpha}$ the radial impurity pressure gradient. $v_{t h, \alpha}$ is the thermal velocity, i.e. $v_{t h, \alpha}=\sqrt{T_{\alpha} / m_{\alpha}}$ with $T_{\alpha}$ being the species temperature and $m_{\alpha}$ the species mass, $Z_{\alpha}$ is the charge of the impurity species, $\tilde{\Phi}$ is the poloidally varying potential where the perturbation is small compared to the equilibrium potential. $\nu_{\alpha i}$ is the collision frequency between the impurities and the main ions, $u_{\|, \alpha}$ and $u_{\|, i}$ are the parallel velocities of the impurity and the main ion species, respectively, and $\Delta Q_{\|}$is the parallel heat flow differential between main ions and impurities, which is proportional to the ion temperature gradient. The terms of equation (6) (from left to right) correspond to the poloidal centrifugal force, the toroidal centrifugal force, the pressure and electric force and the friction force.

The impurity species is assumed to be a trace impurity with $Z_{\alpha} n_{\alpha} \ll n_{i}$, where $n_{i}$ is the main ion density [23]. Furthermore, it is assumed that $T_{\alpha} \approx T_{i}, T_{i}$ being the main ion temperature, since the time needed for an impurity to equilibrate to the main ions is short compared to local transport time scales [30]. For the fluid model the measured ion temperature, electron density, impurity density and the poloidal and toroidal impurity rotation velocity profiles measured at the LFS are used as input. Using the fluid approach, it has been shown through analytic and numerical simulations [23] that the observed flow structure arises due to the interplay between all forces. The friction force plays a dominant role, however, towards the separatrix, where the poloidal impurity rotation reaches its maximum and becomes supersonic (i.e. the Mach number associated with $v_{\theta, \alpha} B / B_{\theta}$ approaches one), the poloidal centrifugal force gives a significant contribution.

In addition, a kinetic approach was used and the experimental data were compared to simulations performed with the drift-kinetic particle code HAGIS [24]. This also enables a comparison between the conventional fluid model and the more comprehensive, kinetic description provided by HAGIS that includes the effects due to finite orbit sizes. The extended version of HAGIS includes a Monte-Carlo pitch angle collision model [25] that enables the calculation of neoclassical transport based on a $\delta f$ method. Here, the unperturbed distribution function $f_{0}$ is a Maxwellian with constant density and temperature on a flux surface, while the perturbed part of the distribution function, $\delta f$, is represented by marker particles which are followed along their orbits and the collisions are modelled by a Monte-Carlo procedure. Simulations of plasmas with a single trace impurity were performed with HAGIS that uses the measured LFS electron density and temperature, ion temperature, impurity ion density and the radial electric field $\left(E_{r}\right)$ profile as input.

In the following, the flow structure resulting from the formulation presented in [23] is 


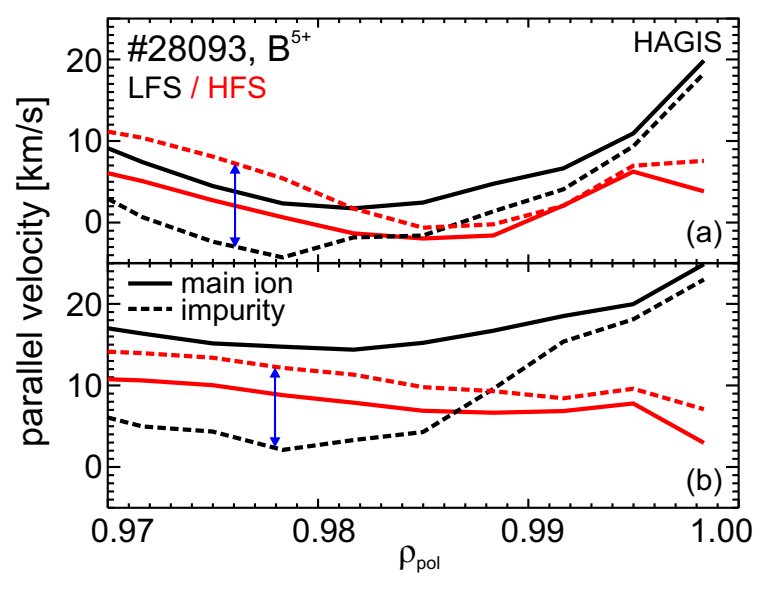

Figure 6: HAGIS simulations of the parallel velocities of the main ions (solid line) and impurities (dashed line) at the LFS in black and HFS in red: (a) using the measured $E_{r}$ profile at the LFS as evaluated from the radial force balance of $\mathrm{B}^{5+}$ and $\nu_{\alpha i}$ calculated using $n_{i}=n_{e}$, (b) using a decreased collision frequency and an $E_{r}$ profile that has been modified within the experimental uncertainties such that the resulting parallel impurity flow at the LFS is similar to the measured profile.

compared to simulations performed with HAGIS [26] and to experimental measurements. First the main ion dynamics is simulated with HAGIS and the resulting profiles (rigid body rotation of the main ions $\omega_{i}$ and main ion poloidal rotation $v_{\theta, i}$ ) are used as input for both models. Then the impurity dynamics is simulated and the resulting flow profiles of both the fluid and the kinetic model are compared to each other.

The HAGIS simulations were performed with an input $E_{r}$ that is derived from the CXRS measurements using the radial force balance of $\mathrm{B}^{5+}$. The resulting parallel velocities for the main ions (solid line) and $\mathrm{B}^{5+}$ impurities (dashed line) at the LFS (black) and HFS (red) are shown in figure 6(a). Note that the LFS parallel velocity of $\mathrm{B}^{5+}$ is too low compared to the actual measured values. An additional simulation was performed with HAGIS in which the input $E_{r}$ was slightly modified (within the experimental uncertainties) and the collision frequency between impurities and main ions, $\nu_{\alpha i}$, was decreased by a factor of 1.5 compared to the nominal collision frequency, that is evaluated using $n_{i}=n_{e}$ ( $n_{i}$ and $n_{e}$ being the main ion and electron density, respectively), such that the resulting LFS parallel impurity flow is similar to the measured value. For the nominal collision frequency (see figure 6(a)) the friction force dominates close to the separatrix $\left(\rho_{\text {pol }}>0.985\right)$ and tends to push the impurity parallel flow towards the main ion parallel flow. For a comparison, the parallel flows of both impurities and main ions at the LFS and HFS are shown in figure 6(b) for the case with a slightly modified $E_{r}$ and collision frequency. Note that in both cases the LFS parallel flow of the main ions (solid lines) is higher than at the HFS, contrary to the parallel impurity flows (dashed lines). This implies that the impurities are dragged by the main ions, however, parallel impurity dynamics can emerge depending on the interplay between the forces in the 


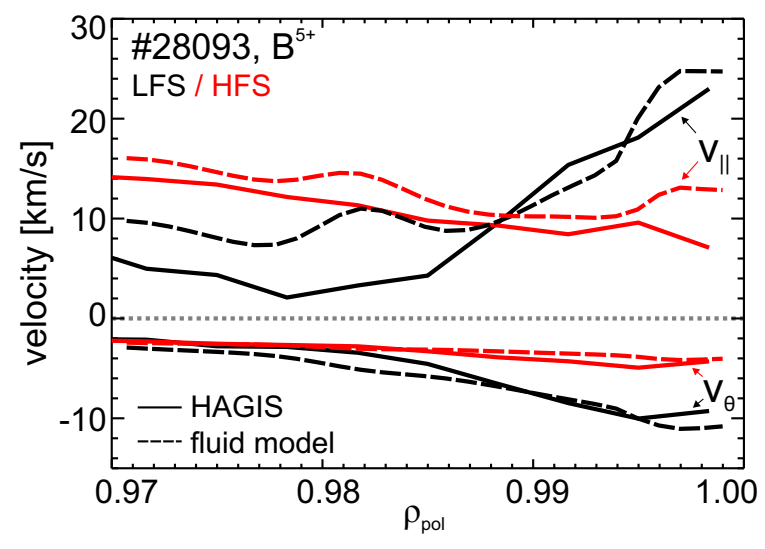

Figure 7: Comparison between HAGIS (solid lines) and fluid model (dashed lines): simulated parallel and poloidal flows at the LFS (black) and HFS (red).

parallel momentum balance.

Figure 7 shows a comparison of the simulated flow profiles resulting from the fluid and the kinetic approach when the same input parameters are applied. In this case, the main ion parameters $\left(\omega_{i}\right.$ and $\left.v_{\theta, i}\right)$ as simulated with HAGIS are used as input and the impurity dynamics is then modelled. Good agreement is obtained between both models demonstrating a consistency between the fluid and the kinetic model.

Figure 8 shows the asymmetry factor, defined as the ratio of the HFS and LFS impurity density $n_{\alpha}^{\text {HFS }} / n_{\alpha}^{\text {LFS }}$, as evaluated using the measured profiles in black, using KN1D in blue (dotted line) and green (dashed-dotted line), and resulting from the simulations using the fluid approach (red, solid line) and HAGIS (purple, dashed line). For better clarity the uncertainties are shown only at distinct radial positions. Within the experimental uncertainties, the simulated asymmetry factors agree qualitatively with the measured one. The maximum of the asymmetry factor is approximately at the same position

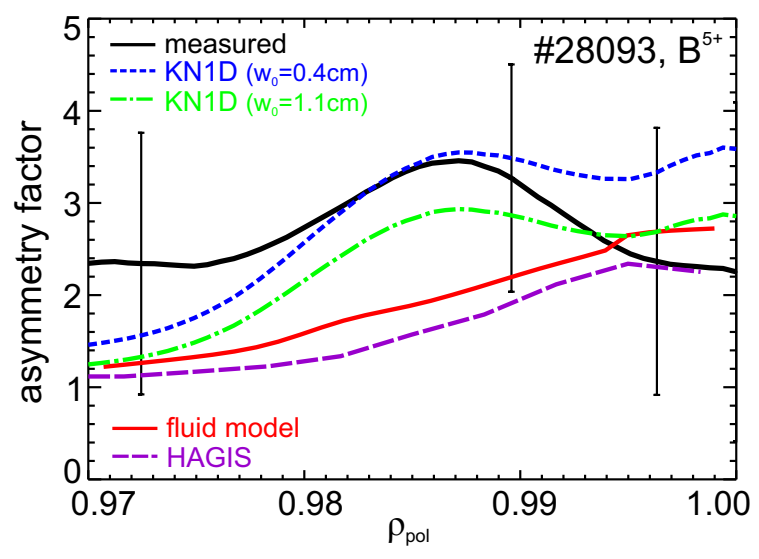

Figure 8: Asymmetry factors resulting from the measured data of $\mathrm{B}^{5+}$ and $D_{\alpha}$ (black), derived from KN1D (blue and green), from the fluid approach (red) and from HAGIS (purple). For better clarity the uncertainties are shown at distinct radial positions. 


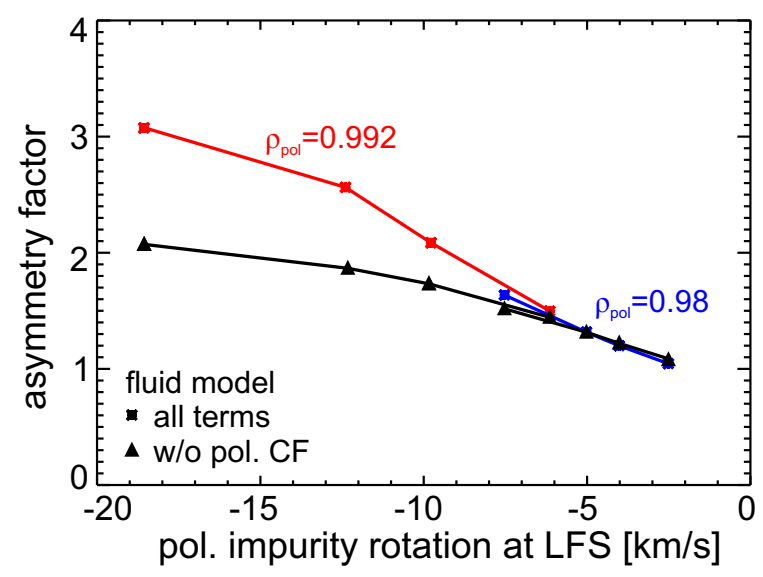

Figure 9: Asymmetry factor versus LFS poloidal impurity rotation velocity.

where the poloidal impurity flow is maximal and becomes super-sonic, in qualitative agreement with [19]. Figure 9 shows the simulated asymmetry factor as a function of the poloidal impurity rotation velocity at the LFS at two radial positions $\left(\rho_{\text {pol }}=0.98\right.$ and 0.992). For higher poloidal impurity flows the asymmetry factor increases and the contribution arising from the poloidal centrifugal force becomes more important.

Compared to the measurement, the separation of the parallel impurity flows and the difference in magnitude of the poloidal flows are modelled qualitatively. However, the difference between the measured HFS and the LFS parallel impurity flows is a factor of $\sim 2$ larger than in the simulations. To obtain the large separation in the parallel impurity flow as observed in the experiment, a parameter scan in the rigid main ion rotation velocity $\omega_{i}$ was performed, while the impurity flows measured at the LFS were used as input [23]. The measured impurity flows are reconstructed quantitatively when $\omega_{i}<0$ and a finite poloidal main ion flow of $\sim 2 \mathrm{~km} / \mathrm{s}$ emerges. The condition $\omega_{i}<0$ means that $E_{r}$ is larger than $\nabla p_{i} / e n_{i}$, but only larger by maximal $2-3 \mathrm{kV} / \mathrm{m}$. Note that though the main ion pressure gradient term is the dominant contribution to $E_{r}[30,40]$ such a small difference is well within the experimental uncertainties. The main ion parallel flow at the HFS is now higher than at the LFS, i.e. contrary to the situation in figure 6 . Due to friction the impurities tend to follow the main ions. Near the separatrix the poloidal centrifugal term increases (due to an increasing $v_{\theta, \alpha}$ ) and contributes to the total shape of the parallel impurity flows (see figure 10). Figure 10 shows a comparison between the measured and simulated parallel impurity flows, where the blue curve represents the modelled profile when the poloidal centrifugal term is neglected. This shows that the poloidal centrifugal force arising from the poloidal impurity flow can have a significant contribution in the parallel force balance.

The fact that a poloidal main ion flow of $\sim 2 \mathrm{~km} / \mathrm{s}$ is needed to explain the measured parallel impurity flows is consistent with neoclassical theory. Figure 11 shows the poloidal main ion flow as derived from the fluid model and in comparison the calculated profiles using the neoclassical code NEOART [41]. NEOART calculates the collisional transport coefficients, which represent the sum of a classical, a Pfirsch-Schlüter and a 


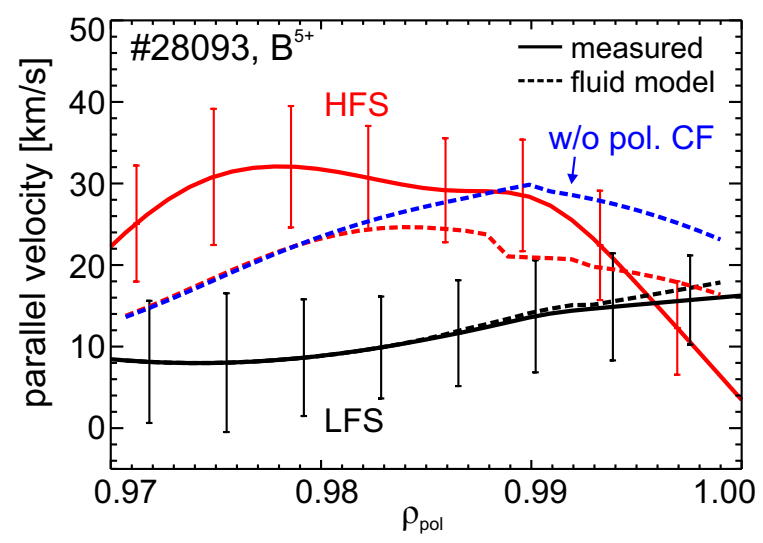

Figure 10: Comparison of the measured and simulated parallel impurity velocities at the LFS and HFS: Here the impact of the poloidal centrifugal force on the resulting flow structure is visible close to the separatrix.

banana plateau term [41, 42], for a given number of impurities and includes collisions between all species. The red profile of figure 11 shows the calculated poloidal rotation profile of the main ions when boron is used as the only trace impurity. The neoclassical prediction shown in blue includes boron, carbon, helium, oxygen and tungsten as impurity species, resulting in an effective charge of $Z_{\text {eff }} \approx 1.6$ at the pedestal top which is typical for an H-mode plasma at AUG [43]. Simulating a multi-species plasma shows that $v_{\theta, i}$ increases from $\sim 1$ to $\sim 2 \mathrm{~km} / \mathrm{s}$. The error bars on the blue profile resemble the uncertainty in the measured $T_{i}$ profile.

The results presented here show that in the edge transport barrier the impurity density is not a flux function and accumulates at the HFS, mainly due to the fricton force. However, also the non-linear inertial term emerging from the poloidal impurity flow can become important, especially in the region where the poloidal Mach number approaches

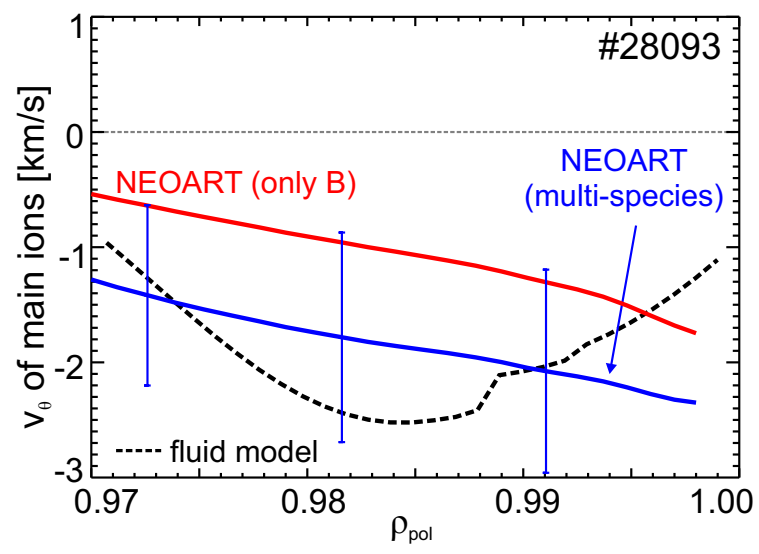

Figure 11: Simulated poloidal rotation of the main ions derived from the fluid model in black (dashed line) and NEOART (red and blue, solid lines). The effect of the presence of multiple impurity species in the plasma $\left(\mathrm{Z}_{\mathrm{eff}} \approx 1.6\right)$ is shown in blue. 
unity, i.e. close to the separatrix [23]. The fact that the impurity density has a poloidal dependence has an impact on the total flow on a flux surface and is responsible for the features of both the toroidal (parallel) and poloidal flows, as observed in experiment.

\section{Impact on impurity transport}

Previous studies on the impurity transport in the edge transport barrier of AUG [2] showed that the experimentally determined radial diffusion coefficients, $D$, and convective velocities, $v$, of light impurities such as $\mathrm{He}^{2+}, \mathrm{C}^{6+}, \mathrm{Ne}^{10+}$ and $\mathrm{Ar}^{16+}$ are well described by neoclassical transport coefficients. Impurity transport analyses based on the evaluation of soft X-ray radiation $[44,45]$ also showed that the transport coefficients of $\mathrm{Ne}$ and $\mathrm{F}$ are at the neoclassical level at the plasma edge. All these studies assumed a constant impurity density on the flux surfaces and the question arises whether a poloidal impurity density asymmetry has an effect on the particle transport.

Due to the redistribution of the impurities along the poloidal arc the friction between main ions and impurities is reduced and thus, the neoclassical particle flux has a non-linear dependence on the temperature and density gradients $[46,6,7]$. If the gradients are sufficiently steep the neoclassical flux can significantly decrease, however, the classical flux increases such that the total flux continues to increase [46].

The neoclassical drift parameter has a $Z$-dependence, i.e. it increases with the local charge of the impurity, since the drift velocity is proportional to the diffusion coefficient multiplied by the ratio of the charge numbers between impurity and main ion. We determined the effect of the impurity density asymmetry on the neoclassical transport, in particular the $Z$ dependence of the drift parameter $v / D$, with simulations using HAGIS for different impurity species. The drift velocity $v$ and the diffusion coefficient $D$, which are determined by the relation $\Gamma=-D d n / d r+n v$, with $\Gamma$ being the flux surface averaged radial particle flux, are obtained from pairs of simulations with different initial impurity density gradients. In the pedestal region, the values of $v / D$ obtained in the stationary

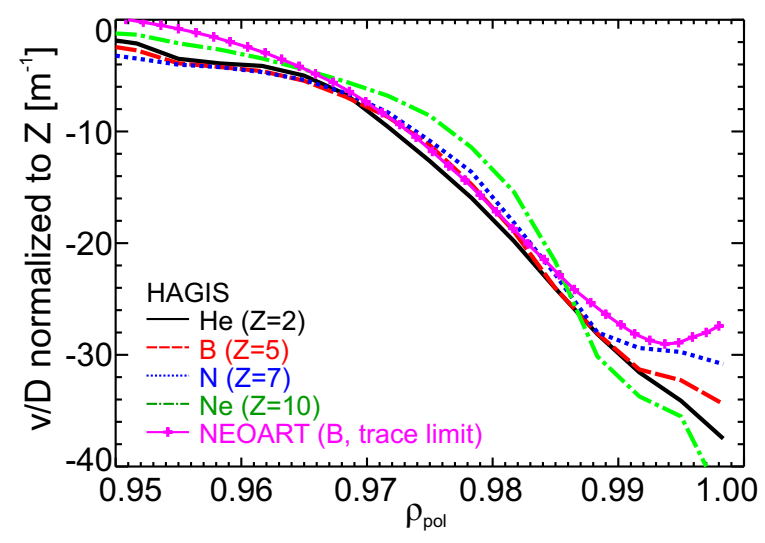

Figure 12: Drift parameter $v / D$ divided by the charge number $Z$ for different impurity species $\left(\mathrm{He}^{2+}, \mathrm{B}^{5+}, \mathrm{N}^{7+}\right.$ and $\left.\mathrm{Ne}^{10+}\right)$ simulated using HAGIS and NEOART. 


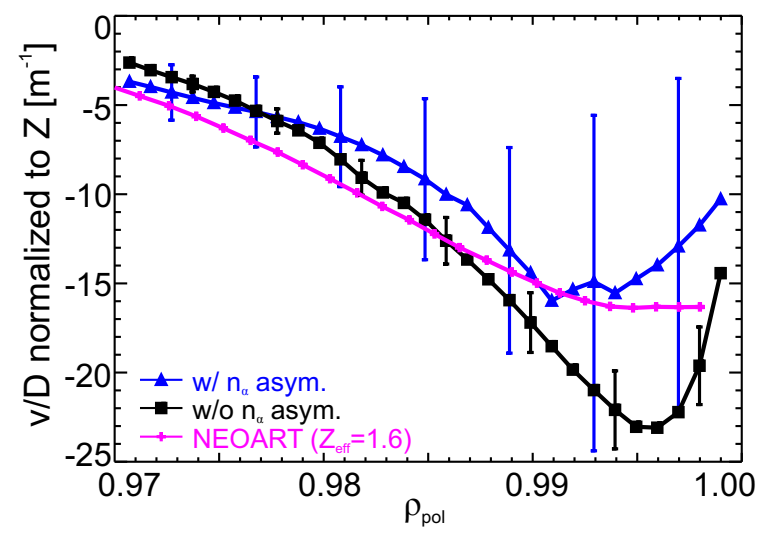

Figure 13: Drift parameter $v / D$ normalized to the charge state $Z=5$ for B simulated with NEOART (magenta crosses), using $Z_{\text {eff }} \approx 1.6$, and evaluated from the measurement assuming no impurity density asymmetry (black squares) and including the poloidal dependence of the impurity density (blue triangles).

state of the simulation are rather inaccurate, since $D$ is very small. Therefore values for $v / D$ were obtained for the early transient phase of the simulation, after the density asymmetry has evolved. Figure 12 shows the radial profiles of $v / D$ divided by the charge number $Z$ for the impurity species $\mathrm{He}^{2+}, \mathrm{B}^{5+}, \mathrm{N}^{7+}$ and $\mathrm{Ne}^{10+}$. Despite the presence of an impurity density asymmetry, the parameter $v / D$ is proportional to the charge number $Z$. This scaling is the same as in the standard neoclassical theory, represented by the simulation obtained with NEOART (magenta line in figure 12) for one trace impurity (boron). NEOART does not account for the existence of a poloidal impurity density asymmetry and hence, the good agreement between the simulated profiles indicates that the asymmetric impurity density profile does not have a big impact on the drift parameter $v / D$.

Using NEOART, the effect of including multiple impurity species $\left(Z_{\text {eff }} \approx 1.6\right.$, as described above) on $v / D$ can be simulated. As shown in figure 13, the drift parameter decreases due to the presence of multiple impurities in the plasma. Comparing this profile to the $v / D$ from the experimental profiles shows good quantitative agreement inside the error bars of the experiment. The drift parameters are evaluated using $v / D=d\left(\ln \left\langle n_{\alpha}\right\rangle\right) / d r_{v o l}$, where $\left\langle n_{\alpha}\right\rangle$ is the flux surface averaged impurity density profile and $r_{v o l}$ the normalized volume flux radius. Here, the black profile has been calculated assuming that the impurity density is constant on a flux surface. The blue profile is evaluated using the flux surface averaged impurity density that accounts for the poloidal dependence of the $\mathrm{B}^{5+}$ density as simulated with the fluid model.

The presence of a poloidal impurity density asymmetry in the edge transport barrier has an impact on the flux surface averaged density and slightly reduces $v / D$. However, the resulting $v / D$ is consistent with the standard neoclassical prediction (shown in magenta in figure 13), in good agreement with [2]. This indicates that despite a poloidal rearrangement of the impurities in the edge pedestal, the impurity particle transport is 
at the neoclassical level.

\section{Summary and Conclusions}

The poloidal flow structure is measured in the edge transport barrier of AUG. Four independent measurements at the outboard and inboard midplane reveal the existence of an asymmetric flow pattern along the flux surface. Inside the edge transport barrier the poloidal impurity flow is strongly sheared in the electron diamagnetic drift direction both at the LFS and HFS. However, the HFS poloidal rotation velocity is about a factor of 1.5-2 lower than at the LFS thus, breaking the expected proportionality between the poloidal flow and the poloidal magnetic field. The toroidal rotation velocity is co-current at both the LFS and HFS, however, the profile exhibits an asymmetric structure. Both the toroidal flow asymmetry and the discrepancy in magnitude of the poloidal flow are explained by an excess of impurity density at the HFS following the postulate of divergence-free flows on a flux surface $[19,20]$. The HFS impurity density profile has been evaluated directly from measurements of the Balmer spectral line and from modelling of the gas puff penetration using the code KN1D. Using both evaluation methods the impurity density at the HFS is found to be up to a factor of 3 higher than at the LFS, demonstrating that in the edge pedestal the impurity density is asymmetric on a flux surface.

Comparison to theoretical predictions [23] based on the parallel momentum balance that includes the poloidal and toroidal centrifugal forces arising due to inertia, the pressure drive, the electric force and the friction force reveals the nature of the parallel impurity dynamics. Here, a fluid model [23] and a drift-kinetic approach using HAGIS [26] have been applied and good agreement between the simulated flows is obtained.

The rearrangement of the impurities along the flux surfaces arises due to the interplay of all terms in the parallel force balance with the friction force playing an important role. However, the inclusion of the poloidal centrifugal force, which has been neglected in previous studies, gives additional contributions especially close to the separatrix where the poloidal impurity flow approaches its maximum. The measured flow structure is reproduced quantitatively by the theoretical prediction when a poloidal main ion flow of $\sim 2 \mathrm{~km} / \mathrm{s}$ emerges in the fluid model, which is in agreement with standard neoclassical calculations. Hence, using the measured impurity flows allows information on the poloidal rotation of the main ions to be obtained indirectly.

Comparing the measured drift parameter evaluated from the flux surfaced averaged impurity density to the neoclassical simulation shows quantitative agreement. This demonstrates that despite the existence of a poloidal impurity density asymmetry the impurity particle transport is neoclassical, consistent with previous studies [2].

In summary, the key features of the experimental data including the shape of the rotation profiles, the poloidal impurity density asymmetry as well as the drift parameter are in line with neoclassical theory. 


\section{Acknowledgements}

The first author would like to thank L. Aho-Mantila, M. Bernert and V. Rohde for very fruitful discussions.

\section{References}

[1] J. D. Callen et al. Phys. Plasmas, 17:056113, 2010.

[2] T. Pütterich et al. J. Nucl. Mater., 415(1):S334-S339, 2011.

[3] W. M. Stacey and D. R. Jackson. Phys. Fluids B, 5:1828, 1993.

[4] B. LaBombard and B. Lipschultz. Nucl. Fusion, 27:81, 1987.

[5] W. M. Stacey. Nucl. Fusion, 27:1213, 1987.

[6] T. Fülöp and P. Helander. Phys. Plasmas, 6:3066, 1999.

[7] T. Fülöp and P. Helander. Phys. Plasmas, 8:3305, 2001.

[8] M. Landreman et al. Phys. Plasmas, 18:092507, 2011.

[9] J. A. Wesson. Nucl. Fusion, 37:577, 1997.

[10] A. G. Peeters et al. Phys. Plasmas, 16:042310, 2009.

[11] F. J. Casson et al. Phys. Plasmas, 17:102305, 2010.

[12] L. C. Ingesson et al. Plasma Phys. Control. Fusion, 42:161, 2000.

[13] Ye O. Kazakov et al. Plasma Phys. Control. Fusion, 54:105010, 2012.

[14] T. Fülöp and S. Moradi. Phys. Plasmas, 18:030703, 2011.

[15] S. Moradi et al. Plasma Phys. Control. Fusion, 53:115008, 2011.

[16] M. L. Reinke et al. Plasma Phys. Control. Fusion, 54:045004, 2012.

[17] T. Pütterich et al., I2.106, this conference. Plasma Phys. Control. Fusion (submitted).

[18] J. Arevalo et al. Conf. Proceedings of the $40^{\text {th }}$ EPS Conference on Plasma Physics, O4.101, http://ocs.ciemat.es/EPS2013PAP/pdf/04.101.pdf, Espoo, 2013.

[19] K. D. Marr et al. Plasma Phys. Control. Fusion, 52:055010, 2010.

[20] T. Pütterich et al. Nucl. Fusion, 52:083013, 2012.

[21] R. M. Churchill et al. US-EU Joint Transport Task Force Workshop, Santa Rosa, California, 2013.

[22] R. J. Fonck et al. Phys. Rev. A, 29(6):3288, 1984.

[23] E. Fable et al. (to be submitted), 2013.

[24] S. D. Pinches et al. Comp. Phys. Commun., 111:133, 1998.

[25] A. Bergmann et al. Phys. Plasmas, 8:5192, 2001.

[26] A. Bergmann et al. (to be submitted), 2013.

[27] E. Viezzer et al. Rev. Sci. Instrum., 83:103501, 2012.

[28] R. M. Churchill et al. Rev. Sci. Instrum. (accepted for publication), 2013.

[29] T. W. Morgan et al. Conf. Proceedings of the $37^{\text {th }}$ EPS Conference on Plasma Physics, ECA VOl. 34A, P5.122, http://ocs.ciemat.es/EPS2010PAP/pdf/P5.122.pdf, Dublin, 2010.

[30] E. Viezzer et al. Nucl. Fusion, 53:053005, 2013.

[31] T. Pütterich et al. Phys. Rev. Lett., 102:025001, 2009.

[32] F. L. Hinton and R. D. Hazeltine. Rev. Mod. Physics, 48(2), 1976.

[33] S. P. Hirshman and D. J. Sigmar. Nucl. Fusion, 21(9):1079, 1981.

[34] H. P. Summers. ADAS User Manual 2.6. http://www.adas.ac.uk/manual.php, 2004.

[35] E. Viezzer et al. Plasma Phys. Control. Fusion, 53:035002, 2011.

[36] B. LaBombard. KN1D User Manual. http://www.psfc.mit.edu/ labombard/KN1D_Source_Info.html, 2001.

[37] L. Aho-Mantila et al. Nucl. Fusion, 52:103007, 2012.

[38] R. Dux et al. Conf. Proceedings of the $39^{\text {th }}$ EPS Conference on Plasma Physics, P2.049, Stockholm (Sweden), 2012. 
[39] B. Geiger et al. Plasma Phys. Control. Fusion, 53:065010, 2011.

[40] R. M. McDermott et al. Phys. Plasmas, 16:056103, 2009.

[41] A. G. Peeters et al. Phys. Plasmas, 7(1):268, 2000.

[42] R. Dux and A. G. Peeters. Nucl. Fusion, 40(10):1721, 2000.

[43] S. K. Rathgeber et al. Plasma Phys. Control. Fusion, 52:095008, 2010.

[44] T. Sunn Pedersen et al. Nucl. Fusion, 40:1795, 2000.

[45] R. Dux. Fus. Sci. Technol., 44:708, 2003.

[46] P. Helander. Phys. Plasmas, 5:3999, 1998. 